\title{
El sentimiento kantiano de respeto como núcleo normativo en investigaciones sociales en contextos de vulnerabilidad
}

\section{The Kantian sense of respect as a normative core in social research in contexts of vulnerability}

JOSÉ FÉLIX LOZANO AGUILAR ${ }^{1}$ (Universitat Politècnica de València)

Artículo recibido: 5 de septiembre de 2021

Solicitud de revisión: 28 de octubre de 2021

Artículo aceptado: 9 de febrero de 2022

Lozano Aguilar, José Félix (2022). El sentimiento kantiano de respeto como núcleo normativo en investigaciones sociales en contextos de vulnerabilidad. Recerca. Revista de Pensament i Análisi, 27(2), pp. 1-22. doi: http://dx.doi.org/10.6035/recerca.6119

\section{Resumen}

La investigación en ciencias sociales, sea observacional o de intervención, se enfrenta a situaciones donde la dignidad de las personas participantes y el respeto a las prácticas culturales pueden verse seriamente afectadas. El objetivo de este artículo es doble. En primer lugar, vamos a reflexionar sobre los retos éticos a los que se enfrenta la investigación en ciencias sociales, con especial consideración a las investigaciones en contextos de vulnerabilidad. En segundo lugar, propondremos el sentimiento kantiano de respeto como marco normativo para orientar la investigación en ciencias sociales.

Palabras clave: ética de la investigación, respeto, vulnerabilidad, imperativo categórico.

\section{Abstract}

Social science research, whether observational or interventional, is confronted with situations where the dignity of participants and respect for cultural practices can be seriously affected. The aim of this article is twofold. Firstly, we will reflect on the ethical challenges facing social science research with special consideration of research in contexts of vulnerability. Secondly, we will propose the Kantian sense of respect as a normative framework to guide social science research.

\footnotetext{
${ }^{1}$ jlozan@dpi.upv.es
} 
Key Words: Research ethics, Respect, Vulnerability, Categorical imperative.

\section{INTRODUCCIÓN}

Cuando hablamos de ética de la investigación, inmediatamente se piensa en la barbarie de los experimentos nazis (Spitz, 2005), en el experimento de Tuskegee (Berche y Lefrère, 2010), en la declaración de Núremberg, la declaración de Helsinki y los principios del informe Belmont. La preocupación por la ética en la investigación biomédica es urgente y necesaria y está bien justificada.

Sin embargo, no es tan frecuente ni inmediata la reflexión sobre la ética en la investigación en ciencias sociales. Pareciera que, al no ser tan invasiva ni afectar tan directamente a la salud y el dolor de las personas, no es tan relevante, pero esto es un error. La investigación sobre la intimidad de las personas, sobre sus hábitos sociales, sobre sus prácticas culturales, sobre sus creencias y sentimientos puede ser tan invasiva y tan dolorosa como la investigación biomédica. Los experimentos de Stanley Milgram (1974) y de Philip Zimbardo (2007), entre otros, son dos ejemplos extremos de esa necesidad.

Además de los temas a los que se enfrentan las investigaciones en ciencias sociales, es importante destacar el contexto en el que, en muchas ocasiones, se realiza la investigación. Mientras que la mayoría de investigaciones biomédicas se realizan en entornos controlados (laboratorios, hospitales, etc.), las investigaciones sociales se dan en contextos incontrolables y, en ocasiones, con poblaciones vulnerables para quien los riesgos potenciales a corto, medio y largo plazo son muy graves. Los desafíos éticos de investigaciones sobre pobreza, marginación, violencia política, bienestar psicológico, etc., son enormes y demandan un gran compromiso ético por parte de los investigadores.

Responder a estos retos no es fácil y, como reconocía la Academy of Social Sciences en el 2017, la aplicación de los modelos de ética de la investigación, basados en códigos de ética que tanto éxito han tenido en las ciencias biomédicas, no han tenido el mismo resultado en ciencias sociales.

While these codes are often treated as if they were universally applicable, social scientists have demonstrated that they have been shaped by the particular circumstances and contingencies of biomedical research on human subjects (Dingwall, Iphofen, Lewis, Oates y Emmerich, 2017: 1). 
Coincidimos con Morris (2015) cuando afirma que aún cuando los investigadores en general aceptan la idea de una supervisión ética, hay una creciente preocupación por la excesiva burocratización de los comités de ética de la investigación y serias dificultades para la aplicabilidad de los modelos de supervisión ética de la biomedicina, especialmente cuando se refiere a métodos cualitativos o etnográficos.

Compartimos esta crítica, pero no la idea de que las directrices internacionales como el informe Belmont, el ASA Code of Ethics o los comités de ética de la investigación no son válidas para las investigaciones sociales porque se centran en el bienestar individual (Emanuel y Weijer, 2005) y que esto supone una institucionalización de la ideología individualista que ha conducido a la discriminación, la opresión y la alienación de otras culturas (Cragoe, 2019). El principio ético de respeto a la dignidad y la autonomía de la persona es el eje de la ética de la investigación tanto en ciencias biomédicas como en ciencias sociales y en humanidades.

La orientación ética en la investigación en ciencias sociales es urgente y cada vez más necesaria. Nuevos desafíos vinculados al manejo de datos, al acceso a la privacidad o a los avances en inteligencia artificial que permiten establecer perfiles de personalidad y comportamiento son de urgente reflexión ética.

El objetivo de este artículo es justificar el sentimiento kantiano de respeto como el principio normativo que debe orientar las acciones y las decisiones en la investigación social en contextos de vulnerabilidad. Comenzaremos presentando los desafíos y las especificidades que supone la investigación en ciencias sociales en contextos de vulnerabilidad. Continuaremos presentando el sentimiento kantiano de respeto y sus rasgos esenciales que lo hacen, a nuestro juicio, el principio ético esencial en investigaciones sociales. En tercer lugar, y como conclusiones, presentaremos las implicaciones prácticas de este sentimiento kantiano de respeto a las prácticas de investigación social en contextos vulnerables.

\section{INVESTIGAR EN CONTEXTOS VULNERABLES: DESAFÍOS ÉTICOS}

Como nos recordaba Habermas, las ciencias histórico hermenéuticas aspiran a la comprensión de sentido y a orientar la acción en base a esa comprensión (Habermas, 1968). El interés práctico del conocimiento desarrollado por las ciencias sociales es analizar la realidad social con la pretensión de com- 
prenderla y poder orientar la acción en vista a la emancipación del sujeto. Así, el interés cognitivo de las ciencias sociales es emancipatorio. Esta afirmación es especialmente relevante cuando la referimos a investigaciones sociales en contextos de vulnerabilidad donde las personas sufren opresión económica, política, cultural y social.

Como hemos apuntado en la introducción, un aspecto especialmente central en investigaciones sociales es el contexto en el que se realizan. Aunque determinadas investigaciones sociales, especialmente en psicología social, se realizan en laboratorios o en otros entornos controlados, la mayor parte de la investigación social se realiza en contextos incontrolados. Y de especial riesgo son las investigaciones en contextos de vulnerabilidad. Las investigaciones sobre temas de discriminación, pobreza, opresión política, dominación cultural, etc., son imprescindibles para luchar contra la injusticia en el mundo. Este tipo de investigaciones son especialmente arriesgadas por la asimetría de poder y la situación de riesgo para la integridad física y psicológica de las personas sujetos de estudio e incluso en ocasiones para los investigadores. ${ }^{2}$

\subsection{Vulnerabilidad: definición y tipología}

En estas investigaciones la clarificación del concepto de vulnerabilidad y su caracterización es esencial. Como recuerdan Schroeder y Gefenas (2009), el concepto de vulnerabilidad se enfrenta a dos desafíos. Por un lado, sigue sin haber una definición precisa y el concepto es vago; y, por otro lado, las categorías de población vulnerable se han extendido tanto que prácticamente todo el mundo es vulnerable. La consecuencia, según las autoras, es que la protección a la población genuinamente vulnerable se está diluyendo.

Según la escueta definición del diccionario de la RAE, vulnerabilidad es: «Que puede ser herido o recibir lesión física o moralmente». Esta definición se centra en aspectos externos de la vulnerabilidad, mientras que la definición del Council for International Organizations in Medical Sciences (2016) se centra en el aspecto interno de la vulnerabilidad al definirla como: «la falta de capacidad para proteger tus propios intereses».

Por nuestra parte, aquí vamos a seguir la definición de vulnerabilidad propuesta por Doris Schroeder y Eugenijus Gefenas: «To be vulnerable means to

\footnotetext{
${ }^{2}$ Especialmente dramático fue el asesinato de Giulio Regini en Egipto en el 2016 (https://www.bbc.com/news/world-middle-east-55266449, consultado el 29 de junio de 2021).
} 
face a significant probability of incurring an identifiable harm while substantially lacking ability and/or means to protect oneself» (Schroeder y Gefenas, 2009: 117). Esta definición combina los elementos internos y externos del fenómeno de vulnerabilidad: la fragilidad de la condición humana que nos sitúa ante la posibilidad de sufrir daño y la potencial falta de capacidad de protegernos a nosotros mismos.

Referido específicamente a la ética de la investigación, podemos identificar seis tipos de vulnerabilidad: cognitiva, jurídica, deferencial, médica, posicional y estructural (Kipnis, 2001).

- La vulnerabilidad cognitiva se refiere a la capacidad del sujeto para decidir si quiere o no participar en la investigación y aquí se incluyen tanto personas con enfermedades mentales, demencia o inmadurez, como personas con importantes déficits educativos.

- La vulnerabilidad jurídica se presenta cuando las personas que participan en la investigación están bajo la autoridad de otra persona (prisioneros, militares, estudiantes, menores, etc.). Esta posición de sometimiento a la autoridad jerárquica formal puede limitar seriamente la libertad del sujeto para decidir.

- La vulnerabilidad deferencial o de adhesión es la coacción subjetiva que sienten las personas por la presión de sus compañeros, amigos, familiares o miembros de la comunidad cultural a la que pertenecen. Los investigadores que trabajan en contextos culturales deben entender las presiones culturales y el poder social al que están sometidos los participantes en la investigación.

- La vulnerabilidad médica es la que hace referencia a la situación de las personas con graves problemas de salud para los cuales no hay remedios satisfactorios. Esta vulnerabilidad es especialmente grave ya que la situación de falta de alternativas, el dolor físico y la desesperación hacen que las personas estén dispuestas a aceptar riesgos más allá de lo razonable.

- La vulnerabilidad posicional o de asignación hace referencia a la situación de privación material (dinero, casa, cuidados médicos...) en la que se encuentran las personas. Esta situación de privación hace que las pequeñas recompensas y las compensaciones sean un gran incentivo para participar en la investigación.

- Y, finalmente, la vulnerabilidad estructural hace referencia a la infraestructura material y legal que existe para llevar a cabo la investigación. Puede ocurrir que no existan los recursos económicos, políticos, legales, regulativos e institucionales básicos para garantizar que los sujetos no están sometidos a altos niveles de riesgo. 
Todas ellas suponen una importante limitación al principio ético básico del consentimiento libre e informado, y una situación de riesgo de manipulación de los sujetos participantes. El imperativo ético kantiano de tratar a las personas como fin en sí mismos y no solo como medio está en peligro en estas situaciones.

En la investigación en ciencias sociales en contextos vulnerables suele darse una superposición de vulnerabilidades y las personas tienen serias limitaciones en su capacidad de decisión para participar o no en la investigación fruto de múltiples tipos de vulnerabilidad. Tres situaciones especialmente graves son las que tienen que ver con investigar en países o regiones empobrecidas, en regímenes totalitarios (Glasius et al., 2018) y en situaciones de emergencia humanitaria (Dittmer y Lorenz, 2018). Dada la complejidad del tema y la limitación de espacio, aquí nos vamos a centrar solo en los desafíos éticos de la investigación en ciencias sociales en contextos de vulnerabilidad estructural. Esta vulnerabilidad estructural se manifiesta en dos niveles fundamentales: la falta de recursos y la debilidad institucional.

La falta de recursos materiales afecta tanto a los investigadores como a los sujetos que participan en la investigación. Por una parte, los investigadores pueden carecer de los recursos materiales y humanos para desarrollar las investigaciones con garantías. Por ejemplo, pueden carecer de un laboratorio con las adecuadas medidas de seguridad, de unos técnicos suficientemente formados o de medidas de protección adecuadas. Por su parte, las condiciones de pobreza de los participantes pueden llevarles a aceptar situaciones de alto riesgo y sus condiciones socioeducativas (por ejemplo, analfabetismo) pueden significar que desconozcan sus derechos y cómo reclamarlos.

La debilidad institucional supone la falta de procedimientos, protocolos o instituciones adecuados para supervisar las investigaciones y garantizar los derechos de los participantes. La ausencia o el funcionamiento deficiente de los comités de ética de la investigación, la falta de mecanismos independientes de revisión e incluso la falta de regulación o la injerencia política son factores que suponen un alto riesgo para una investigación responsable.

\subsection{Investigar en países empobrecidos y el riesgo de Ethics Dumping}

La cooperación internacional en investigación es claramente positiva y la universalidad del conocimiento ha sigo un rasgo distintivo desde el origen de la búsqueda del saber. El intercambio de ideas, experiencias y metodologías puede ser especialmente fructífero entre países de diverso nivel económico, 
social y de avance científico. Mediante la colaboración científica los países de ingresos medios y bajo (LMIC) pueden recibir fondos, formación y tecnología que de otra forma no recibirían. Por su parte, los países de ingreso alto (HIC) pueden acceder a material, contextos y conocimientos diferentes. Sin embargo, esta colaboración puede verse muy desfavorecida por la práctica de lo que la Unión Europea denominó en el 2014 Ethics dumping.

Doris Schroder y colaboradores definen el ethics dumping como:

Ethics dumping involves the export of research which would be severely restricted or not permitted in HICs to LMICs, where ethical review processes, compliance structures and follow-up mechanisms might not be as well-resourced or supported (Schroeder et al., 2019: 24).

Esta práctica consiste en trasladar a terceros países investigaciones que la regulación europea no permitiría o dificultaría debido a las implicaciones éticas de dicha investigación. Investigación con animales, con sustancias peligrosas, manejo de datos o investigaciones médicas de alto riesgo son algunos de los ejemplos que se han identificado en las últimas décadas (Schroeder, Cook, Hirsch y Fenet, 2018). Un ejemplo paradigmático de este tipo de dumping es el caso del investigador Juan Carlos Izpisúa, quien trasladó sus investigaciones con células madre a China. ${ }^{3}$ Aunque la motivación de evitar la regulación legal y los controles éticos no es la única. El dumping ético también se realiza buscando condiciones socioeconómicas y culturales más ventajosas, así como explotar recursos materiales y humanos.

Según Schröder, Chatfield y Muthuswamy (2020), las actitudes y las conductas de los investigadores más extendidas asociadas al dumping ético son las conductas paternalistas, la distribución no equitativa de cargas y beneficios, la conducta cultural inapropiada, los dobles estándares y la falta de transparencia.

- Las conductas paternalistas, donde los investigadores adoptan una actitud de superioridad intelectual y moral hacia sus contrapartes y las personas que participan en sus investigaciones. Las actitudes de «nosotros lo sabemos mejor» o «lo podemos hacer mejor» hacen que se trate a las personas en estos contextos como incapaces de entender lo que se hace y de decidir sobre su participación. Estas actitudes de arrogancia se manifiestan en conductas

\footnotetext{
${ }^{3}$ El objetivo de la investigación es crear quimeras inyectando células de monos en células madre humanas. La finalidad última de la investigación sería la generación de órganos bumanoides en animales que pudieran servir para trasplantes en humanos (Ansede, 2019).
} 
como ignorar o despreciar a los comités de ética de estos países, ignorar sus intereses y conocimientos o no reconocer su aportación a la investigación.

- La distribución inequitativa entre cargas y beneficios. El dumping ético busca obtener unos beneficios a costa de explotar los recursos o la información de los contextos vulnerables sin aportar nada o muy poco a cambio. Esta actitud neocolonial implica la extracción o la utilización de recursos sin considerar los beneficios que la investigación pueda aportar a los individuos o las comunidades locales.

- La conducta cultural inapropiada parte de la falta de sensibilidad hacia las culturas propias de los contextos donde se realiza la investigación. Determinadas actitudes, símbolos o prácticas de los investigadores de los países de altos ingresos pueden ser consideradas ofensivas por parte de las personas de las comunidades locales. Esta falta de sensibilidad puede deberse a una conciencia etnocéntrica o simplemente al desconocimiento y la indiferencia hacia las culturas locales. Ambas son inaceptables.

- La aplicación de dobles estándares hace referencia explícita a la búsqueda de menores exigencias legales y éticas para realizar las investigaciones. Actividades que en Europa, Estados Unidos o Japón estarían prohibidas o altamente reguladas, se trasladan a estos países con intención de evitar esas prohibiciones o controles. Esta práctica es especialmente negativa porque normalmente se refiere a investigaciones de riesgo para las personas, los animales o el medio ambiente, con lo cual, ante un posible error o accidente, las consecuencias para las comunidades locales pueden ser muy graves.

- Falta de transparencia respecto a los fines de la investigación o a las fuentes de financiación. En muchas investigaciones en LMIC ni los participantes ni, en muchas ocasiones, las autoridades tienen un conocimiento real de cuál es el objetivo final de la investigación, ni quién la está financiando. Esto supone un grave riesgo de instrumentalización de las personas y, en ocasiones, conlleva poner en peligro a los sujetos participantes en la investigación.

Sin duda, estas actitudes y conductas son un problema real que supone, en muchos casos, una violación directa de los derechos humanos y un peligro para el bienestar animal y de la protección del medio ambiente. A nivel general, el mejor antídoto contra el dumping ético es una colaboración intensa, equitativa y transparente entre los investigadores (locales y extranjeros) y las comunidades en las que se desarrolla la investigación (Schroeder, Chatfield, y Muthuswamy, 2020).

El código de conducta global para investigar en entornos de bajos recursos (Global Code of Conduct for Research in Resource-Poor Settings, GCC) es un docu- 
mento especialmente diseñado para luchar contra el dumping ético. Este código, elaborado participativamente por stakeholders de países ricos y de países de ingreso bajo y medio, se aprobó en el 2018 y está construido en base a cuatro grandes valores: equidad, respeto, cuidado y honestidad. Estos valores se concretan en principios, estándares y guías de conducta e ideales.

\section{EL SENTIMIENTO KANTIANO DE RESPETO}

\subsection{Introducción}

El Global Code of Conduct for Research in Resource-Poor Settings (GCC), el Sand Code y la guía para Ethics in Social Science and Humanities de la Comisión Europea (European Commission, 2018) son instrumentos útiles y necesarios para orientar las conductas de los investigadores en contextos vulnerables, pero ni sustituyen la reflexión ética personal del investigador, ni pueden ser entendidos como un procedimiento de aplicación mecánica.

Estos documentos y otros similares, como los códigos éticos de las agencias de cooperación, enfrentan a nuestro juicio dos grandes retos: uno es tener la fundamentación filosófica normativa necesaria para superar el relativismo moral y el segundo es la integración de los valores y los principios en la práctica de investigación concreta.

En el caso concreto del GCC, el valor respeto es uno de los pilares fundamentales del código y los cuatro artículos en que se concreta este valor $(8,9,10$ y 11) son imprescindibles guías de conducta. Y lo mismo puede decirse de la guía de la Comisión Europea Ethics in Social Science and Humanities, donde se afirma categóricamente que hay que «respetar la autonomía», «respetar el medio ambiente» $\mathrm{y}$ «respetar la dignidad humana». El problema, a nuestro juicio, es que dicho valor no está definido con claridad en estos documentos y que su formulación, tanto en el valor como en los principios, es imprecisa y puede generar confusión en situaciones de conflicto moral. Como reconocen Schroeder y colaboradores, en investigaciones sobre determinadas prácticas culturales no se puede identificar respetar con aceptar, como parecen indicar la declaración de Helsinki y los documentos citados anteriormente. Eso nos llevaría a lo que Marcuse llamó la «tolerancia represiva» (Marcuse, 1965) o a un concepto de tolerancia que se ha convertido en ideología posmoderna y que poco tiene que ver con el respeto activo (Lozano y Escrich, 2017). La reflexión sobre lo que supone respetar una práctica cultural es inevitable: 
It is indeed sometimes difficult to strike a balance between dogmatically imposing one's own approach and carelessly accepting human rights violations, but that is the balance researchers should strive for (Schroeder et al., 2019: 23).

Este esfuerzo de reflexión no puede darse sin un sólido bagaje conceptual. $\mathrm{Y}$ aquí es donde nosotros, humildemente, queremos hacer una aportación. Consideramos que el sentimiento kantiano de respeto a las personas como fin en sí mismo puede ser un fundamento sólido para orientar la reflexión y la acción de investigación en contextos vulnerables y marcar los límites entre las prácticas y las conductas culturales que se debe respetar y las que no.

Respeto viene del latín respicere cuya traducción hoy sería 'mirar atentamente algo' o 'volver a mirar', por lo que podemos afirmar que una primera definición del concepto tiene que ver con prestar especial atención a un objeto o persona, y sería lo contrario a la indiferencia, la negligencia o la falta de atención.

El concepto de respeto, al igual que la idea de autonomía (Conill, 2016), tiene un protagonismo central en la filosofía moral desde la Ilustración, aunque su historia se remonta a muchos siglos antes. Ya en la poesía griega, en Platón y Aristóteles tiene cierto protagonismo (Giorgini e Irrer, 2017), aunque es cierto que es un concepto de respeto referido a la posición social y vinculado al honor. Alguien merece respeto por la posición social o la familia a la que pertenece.

El concepto ilustrado de respeto es radicalmente diferente, en cuanto considera la autonomía (capacidad de darse normas) como fuente de respeto. El respeto es una expresión de la agencia, de la capacidad de establecer fines, de analizar, deliberar y considerar reflexivamente el objeto, persona o fenómeno a respetar. Este concepto de respeto tiene una dimensión racional esencial. Respetamos a alguien o a algo porque tenemos razones para hacerlo. El respeto no es arbitrario, necesitamos razones para respetar (Darwall, 1977). El respeto, a nivel general, puede dirigirse a instituciones, objetos o personas. Aquí nos vamos a centrar en el respeto hacia las personas, respeto como reconocimiento moral que implica la valoración (estima) de las personas como miembros de la comunidad humana.

\subsection{Sentimiento kantiano de respeto}

El sentimiento de respeto en Kant está asociado a la idea de dignidad y valor en sí mismo, y en su momento supuso la superación de dos concepciones 
tradicionales del respeto: la tradición aristocrática asociada al honor y la concepción instrumental asociada al valor de lo que aporta la persona. Para Kant, la dignidad se identifica con el absoluto valor intrínseco a la persona (Schroeder y Bani-Sadr, 2017). En las páginas finales de La metafísica de las costumbres, Kant afirma que:

El respeto que tengo por otros o que otro puede exigirme es el reconocimiento de una dignidad en otros hombres, es decir, el reconocimiento de un valor que carece de precio, de equivalente, por el que el objeto valorado pudiera intercambiarse (Kant, 1797: 462).

Para Kant, un ser racional se distingue de todo lo demás en la naturaleza por el hecho de que actúa no meramente de acuerdo con la ley, sino de acuerdo con su propia representación o concepción de una ley (Kant, 1785). En la Fundamentación de la metafísica de las costumbres Kant establece una distinción muy importante entre actuar de acuerdo a la ley y por respeto (Achtung) a la ley. Para él, solo la voluntad que se mueve por respeto puro a la ley puede calificarse de buena voluntad. Y este concepto de respeto, como un sentimiento que surge espontáneamente del concepto de razón y se manifiesta en la voluntad de cumplir la ley práctica, no debe entenderse como una emoción que me invade (como podría ser el miedo) sino como un sentimiento generado por la razón:

Lo que yo reconozco inmediatamente para mí como una ley lo reconozco con respeto, y este respeto significa solamente la conciencia de la subordinación de mi voluntad a una ley, sin la mediación de otros influjos en mi sentir (Kant, 1785: BA17). Göbel,

Este concepto de respeto es el a priori de la moralidad. Como reconoce

Respect is the only feeling that is produced by reason, more precisely: by the insight into the superiority of the moral law. Kant also calls it a "self-produced feeling", and as such it is a priori (Göbel, 2017: 161).

En la misma línea apunta Anastasia Berg (2021) cuando afirma que la identidad entre el respeto moral y la determinación de la acción contiene dos elementos: el respeto moral como forma de autoconciencia práctica que supone el reconocimiento de la ley moral y que está intrínsecamente vinculado con la agencia moral y el respeto como la capacidad de receptividad (receptive 
awarness) de determinados rasgos de nuestro entorno y de otras personas y de cómo ellas nos benefician o perjudican. En este sentido, podría decirse que el sentimiento de respeto es el punto de unión entre el sentimiento moral y la conciencia moral.

Como reconoce el profesor Conill,

El sentimiento moral es la capacidad sensible, física, de recibir el impulso racional y transformarlo en motivo; y la conciencia moral es el hecho inevitable, instintivo, que nos mueve a juzgarnos a nosotros mismos conforme a la ley moral en el foro interno (Conill, 2016: 18).

Así entendido, el sentimiento de respeto cumple dos funciones relevantes para la acción moral: por una parte, es la causa o motivo de la acción, aunque no es autónoma ni suficiente en sí misma (función causal), y, por otra parte, constituye el criterio para diferenciar una acción por deber de una acción conforme al deber (función diferenciadora).

En este punto conviene hacer una importante aclaración sobre el respeto como sentimiento. Kant afirma claramente que el sentimiento de respeto es un sentimiento que emerge espontáneamente de la razón. Esto ha generado gran controversia e incluso algunos autores han considerado que Kant era inconsistente en su idea, puesto que parece que la ley moral se fundamenta en la razón, mientras que la acción moral solo es posible desde el sentimiento. En consecuencia, el sentimiento de respeto ha sido calificado como el más controvertido en la filosofía moral kantiana (Berg, 2021) y ha generado dos grandes interpretaciones: las interpretaciones intelectualistas, que minimizan la relevancia del respeto al afirmar que este no es necesario para la determinación de la voluntad de acción moral, y las interpretaciones afectivistas, que consideran que el respeto moral tiene un papel crucial en la motivación de la acción moral. Nosotros compartimos la interpretación de Berg cuando afirma que ambas interpretaciones tienen debilidades sustanciales y que: «a coherent interpretation of Kant's account of moral respect requires recognizing the identity of the conscious determination of the will by the law and the feeling of moral respect» (Berg, 2021: 11). Así, consideramos que el respeto moral es, en primer lugar, la forma de la autoconciencia práctica constitutiva de la agencia práctica y, a la vez, un sentimiento particular que está dirigido a un fin particular condicionado moralmente. Esta capacidad subjetiva de convertir en móvil el deber es un sentimiento moral que no afecta al fundamento de las leyes 
prácticas (al aspecto objetivo) sino solo al efecto subjetivo en el ánimo (Conill, 2016: 17).

Para Kant, este sentimiento no es un poder independiente con sus propios principios, sino es antes una forma de conciencia de cómo los objetos benefician o perjudican el ejercicio de nuestra voluntad. Es decir, es la forma de la conciencia de cómo las cosas fomentan o limitan nuestra libertad, nos fomentan o limitan como seres libres, racionales y corporales. El respeto moral es idéntico a la consciente determinación de la voluntad humana, pero no es el fundamento de determinación, sino que lo es la ley moral misma (Torralba, 2020: 181). Y en la misma línea se manifiesta Schadow cuando reconoce que: «Conciencia moral y sentimiento moral son dos aspectos de la motivación moral, que describen uno y lo mismo: la determinación de la voluntad mediante una ley práctica incondicionada» (Schadow, 2015: 298). O, por utilizar palabras de Dávila, «No sería nada aventurado sostener que los términos sentimiento de respeto a la ley moral y garantía de la existencia de la posibilidad de la libertad en el mundo sensorial son sinónimos» (Dávila, 2011: 149).

\subsection{Del respeto a la ley al respeto a la persona}

El respeto a la ley se convierte en respeto a la persona mediante el imperativo categórico. La fórmula de la humanidad, contenida en la segunda formulación del imperativo categórico, expresa que lo que es un fin en sí mismo debe ser tratado como tal y, puesto que el ser humano es esencialmente un ser libre, debe ser tratado como un ser libre. En este sentido la libertad es la ratio essendi de la ley moral (Sensen, 2011). Esto implica que quien respeta a otro no debe tratarlo como un simple medio, ni degradarlo (deber negativo). Se trata de un deber estricto que se concreta en el deber de respetar al prójimo y no convertirlo en un medio para mis fines. La definición moderna de dignidad, inspirada en Kant, supone la inviolabilidad de todos los seres humanos, lo que supone el derecho de no ser tratado nunca como simple medio, sino siempre como fin en sí mismo (Schroeder, 2008).

Pero este no es el único sentido de respeto que podemos identificar en Kant. Según Stephen L. Darwall (1977), la distinción entre respeto de valoración (appraisal respect) y respeto de reconocimiento (recognition respect) se puede encontrar en Kant. Esta distinción nos permite responder a la difícil pregunta de si las personas merecen ser objeto de respeto por sus acciones o por el mero hecho de ser personas. El respeto de reconocimiento implica otorgar la consideración apropiada a un objeto/sujeto por alguna característica de 
él. En este caso, el respeto de reconocimiento hacia la persona está basado en la capacidad de estas de usar su razón. Por otra parte, el respecto de valoración es valorar a una persona por sus acciones, por los rasgos de esa persona que atribuiríamos a su carácter. En este segundo sentido, respeto tendría sentido similar a admiración o estima positiva; y, siguiendo a Kant, los rasgos de las personas que se hacen merecedoras de respeto son aquellos que son rasgos en tanto que agentes, como seres que podríamos calificar de excelentes personas.

La exigencia de respeto a otras personas consiste, junto a no instrumentarlas, en seguir una máxima de no valorarse a uno mismo por encima de los demás ni considerar que es mejor que los otros. El respeto que debemos a otro es diferente al sentimiento de aprecio. En la misma línea que Darwall (1977), pero yendo más allá, Oliver Sensen afirma que Kant utiliza el concepto de respeto en tres sentidos diferentes: el aprecio que se tiene por los logros de los demás (appraisal respect), el sentimiento moral hacia el imperativo categórico (recognition respect) y la exigencia de seguir la máxima de no valorarse por encima de los demás (commanded respect) (Sensen, 2011). Esta última concepción de respeto implica que cada ser humano debe ser respetado independientemente de su mérito, sea este moral o de otro tipo; incluso el «hombre vicioso» debe ser tratado como un ser humano. Esta exigencia está implícita en el imperativo categórico en su fórmula de la humanidad.

Las implicaciones prácticas del sentimiento de respeto fueron expuestas por Kant en La metafísica de las costumbres, donde desarrolla la tarea de: «[...] desentrañar las condiciones subjetivas para encarnar en la vida cotidiana el principio moral, descubierto en el proceso de fundamentación» (Cortina y Conill, 1989: 37). Allí afirma que los deberes hacia los demás, considerados como personas, son la benevolencia, la filantropía y la gratitud; mientras que los vicios que suponen una falta de respeto a las personas son la soberbia, la calumnia y la burla (Kant, 1797: 465).

Una primera, y más relevante, implicación práctica de la noción kantiana de respeto es tratar a cada persona como un ser individual y eso supone:

(...) atender a cada persona como individuo distintivo y a las realidades concretas de las vidas humanas, y debe implicar la valoración de la diferencia así como de la igualdad y de la interdependencia así como de la independencia (Dillon, 2007: 209).

El respeto requiere la capacidad de juzgar y reflexión racional. La capacidad de juzgar permite el conocimiento y la comprensión de las diferencias (aunque no se compartan), lo que conduce a otra característica importante del 
respeto, el reconocimiento genuino. Un respeto y un reconocimiento que no es puramente formal, ni únicamente racional, sino que incorpora una dimensión emocional. Es lo que Adela Cortina ha llamado respeto activo:

Consiste el respeto activo no solo en soportar estoicamente que otros piensen de forma distinta, tengan ideales de vida feliz diferentes a los míos, sino en el interés positivo por comprender sus proyectos, por ayudarles a llevarlos adelante, siempre que representen un punto de vista moral respetable (Cortina, 1997: 240).

\section{CONCLUSIONES. ORIENTACIONES PARA LA INVESTIGACIÓN SOCIAL EN CONTEXTOS VULNERABLES}

Del sentimiento kantiano de respeto presentado en el apartado anterior podemos extraer unas conclusiones con claras implicaciones para la investigación en contextos vulnerables.

Una situación especialmente difícil para los investigadores es cuando trabajan en contextos culturales diferentes y con cosmovisiones y prácticas culturales muy diferentes a sus ideales y concepciones de justicia. La difícil pregunta que se presenta en ocasiones es si se deben respetar prácticas culturales opresivas (Sen, 2006). A nuestro juicio, la pregunta de si se debe respetar a una persona o respetar a una cultura debe ser contestada de forma clara y precisa. Y, para eso, conviene hacer una distinción entre lo que supone respetar a las personas y respetar a las culturas o a las prácticas cultural.

¿Qué supone respetar una práctica cultural? ¿Supone aceptarla y no intentar cambiarla? No, a nuestro juicio, comprender una práctica cultural es conocer su razón de ser y su evolución, su sentido; justificarla es considerar que esa razón de ser es valiosa, positiva, y universalizable. Aceptarla y no intentar cambiarla es actuar sin pretender interferir en ella. Mientras que comprenderla y justificarla es una actitud reflexiva, aceptarla y no intentar cambiarla es un comportamiento práctico con peligrosas implicaciones prácticas. El proceso ideal de respeto cultural comenzaría por comprender las prácticas, considerar justificados sus principios y - si los principios son justificados- aceptarlas. Pero la realidad es que muchas prácticas culturales son comprensibles, pero no justificables y, en consecuencia, no aceptables. Un ejemplo claro de esto es lo que se ha dado en llamar las harmful traditions o tradiciones dañinas (Erken, 2020). Prácticas como el matrimonio infantil, la ablación del clítoris o el quemado del pecho en las niñas son tradiciones que hacen mucho daño no solo a 
las personas que sufren individualmente esta práctica, sino a toda la comunidad. Ante estas prácticas, el relativismo moral no es una respuesta aceptable.

Nuestra respuesta es que se debe respetar a las personas y que la libertad real de las personas es la esencia de la legitimidad de una práctica cultural (Cortina, 1997; Sen, 2006). Y creemos que una noción específicamente kantiana de respeto está mejor equipada para responder a los desafíos concretos de la investigación en contextos de diversidad cultural. Como ya argumentamos años atrás (Lozano y Escrich, 2017), la respuesta al reto de la diversidad no puede venir ni desde la imposición de estándares internacionales ni desde una adaptación irreflexiva a los contextos.

En este punto conviene hacer un inciso para clarificar nuestra idea de persona y de autonomía. No estamos aquí defendiendo una idea de persona como sujeto independiente de su contexto y guiado solo por sus intereses particulares; ni la idea de un sujeto abstracto fuera de su comunidad cultural, social, política y geográfica en la que vive. La idea de persona que proponemos no es un individuo, sino que es un sujeto que se construye a sí mismo mediante la acción en un contexto concreto (Korsgaard, 2009), que tiene múltiples identidades y que con más o menos reflexión selecciona voluntariamente sus identidades (Sen, 2006).

La idea de identidad práctica propuesta por Korsgaard supone entender que la identidad está vinculada con la acción reflexiva y autoconsciente. Partiendo de la concepción de razón práctica propuesta por Kant, Korsgaard define la identidad práctica como:

[...] a description under which you value yourself and find your life worth living and your action to be worth undertaking. Conceptions of practical identity include such things as roles, and relationships, citizenship, memberships in ethnic or religious groups, causes, vocations, professions and offices (Korsgaard, 2009: 20).

Así, podemos pensar en una identidad práctica concreta como un conjunto de principios, entre los cuales un agente selecciona y prioriza como fuente esencial de su unidad, como un todo coherente. En este sentido, el conjunto de identidades que puedo seleccionar son contingentes y pasan a ser necesarias (para mí) cuando la persona decide asumirlas como fuente de razón y de obligación que guía la acción.

Desde esta idea de persona como agente cuya identidad se construye a sí mismo mediante la acción racional autorreflexiva es desde la que concluimos que se debe respetar a las personas antes que a las prácticas culturales o a las 
comunidades. Y que esta concepción tiene legitimidad universal y consecuencias prácticas globales. A nuestro juicio, la crítica al concepto de autonomía como etnocéntrica no es acertada por dos razones: la primera es que critica un concepto de autonomía que no es el defendido por la tradición kantiana y la segunda es porque una negación de la autonomía práctica o de la agencia conduciría a un nihilismo moral.

Así pues, partiendo de estos dos grandes conceptos de inspiración kantiana - el concepto de persona autónoma y el sentimiento de respeto- y siguiendo los principios de una ética cívica cordial propuestos por Cortina (2007), proponemos que las personas que realizan investigaciones sociales en contextos de vulnerabilidad actúen de acuerdo a los siguientes cinco principios.

- Sensibilidad hacia el sufrimiento y el dolor. Una primera consideración derivada del sentimiento kantiano de respeto es la exigencia moral de sensibilidad hacia los que sufren. "Así pues, es un deber no eludir los lugares donde se encuentran los pobres a quienes falta lo necesario, sino buscarlos» (Kant, 1797: 457). La interpretación de la teoría moral kantiana como fría y «alejada del dolor» es incorrecta y no hace justicia a su interpretación. Como bien reconoce Adela Cortina, filósofa de reconocida tradición kantiana, «Conocemos la justicia no solo por la razón, sino también por el corazón» (Cortina, 2007: 221).

- Respetar la autonomía y la capacidad de las personas de decidir por sí mismas. No instrumentalizar a las personas y superar el paternalismo. El principio de autonomía de la bioética tiene plena vigencia en investigaciones sociales y más en contextos sociales de vulnerabilidad. Las actitudes de instrumentalización y de paternalismo no son aceptables. El concepto kantiano de humanidad exige que se respete la libertad de los sujetos de investigación en todos los casos y que se cuente con ellos en las decisiones que les afectan. Considerar a la persona como interlocutor válido en las decisiones que le afectan es un principio básico derivado del respeto a la autonomía (Cortina, Conill y García-Marzá, 2008; Cortina, 1985, 1986; Habermas, 1983).

- Fortalecimiento de las capacidades de las personas. Dado que las personas en situaciones de vulnerabilidad tienen importantes limitaciones personales y contextuales para el ejercicio de la libertad, se debe empoderar y fortalecer sus capacidades, siempre desde el respeto a su dignidad. Como recordaba Kant en La metafísica de las costumbres: «Hacer el bien, es decir, ayudar a otros hombres necesitados a ser felices, según las propias capacidades y sin esperar nada a cambio, es un deber de todo hombre» (Kant, 1797: 329). Y en 
línea con el enfoque de capacidades defendido por Amartya Sen (1999, 2006) y Martha Nussbaum (200o), la superación de la injusticia y la desigualdad requiere el fortalecimiento de las capacidades de las personas para que lleven la vida que tienen razones para valorar. Toda investigación en contextos vulnerables debe hacer una contribución positiva a la expansión de las libertades reales de las personas en esa situación.

- Distribución equitativa de las cargas y los beneficios, teniendo como referencia intereses universalizables (principio de la justicia distributiva). El principio de compartir con las personas y las comunidades los beneficios obtenidos de la investigación es una exigencia de justicia. Este principio es bien conocido en el trabajo con comunidades vulnerables y se conoce como benefit sharing y tiene por objetivo mejorar la situación de los más desfavorecidos.

- Minimizar el daño hacia los seres sintientes no humanos y trabajar por un desarrollo sostenible. Entender que la investigación genera impactos negativos en el entorno natural y social e intentar reducirlo es fundamental. El principio de responsabilidad por los seres indefensos no humanos y el cuidado del entorno natural es una obligación moral hacia los seres vivos del presente y hacia las generaciones futuras (Cortina, 2010, 2009).

Así pues, y sintetizando, la investigación en contextos vulnerables y culturalmente diversos se enfrenta a dos problemas: el dumping ético y el respeto a prácticas culturales diversas. La violación de derechos fundamentales de los participantes en una investigación debido a su situación de vulnerabilidad es especialmente grave en investigaciones biomédicas. Mientras que los problemas de respecto a las prácticas culturales diversas se da en investigaciones sociales y humanidades. En cualquier caso, esta es una clasificación analítica que nos sirve para comprender mejor el desafío de investigar en contextos de vulnerabilidad. La realidad es que las situaciones concretas suelen presentar casos complejos y desafiantes que requieren capacidad de juicio moral del investigador y sólida fundamentación en principios éticos.

Nuestra humilde aportación en este artículo ha pretendido fundamentar y dar un marco normativo sólido a orientaciones ya reconocidas en la investigación en ciencias sociales. Unas orientaciones que, sin una fundamentación filosófica rigurosa, pierden fuerza y dejan la puerta abierta al relativismo moral y a la indiferencia frente al dolor y la injusticia. Creemos que el sentimiento kantiano de respeto puede aportar esta fundamentación sólida para orientar las acciones de las personas que trabajan en investigación en contextos de vulnerabilidad. 


\section{BIBLIOGRAFÍA}

Ansede, Manuel (2019, 3o de julio). Científicos españoles crean quimeras de humano y mono en China. El País.

Berche, Patrick y Lefrère, Jean Jacques (2010). The Tuskegee Syphilis Study. La Presse Médicale (1983), 39(12), 1324-1329. doi: http://dx.doi.org/10.1016/j.lpm.2 010.03.030

Berg, Anastasia (2021). Kant on Moral Respect. Archiv Fur Geschichte Der Philosophie, 1-31. doi: http://dx.doi.org/10.1515/agph-2019-0022

Conill, Jesús (2016). Disposiciones de la naturaleza humana y autonomía moral en la filosofía práctica de Kant. Revista de Estudios Kantianos, 1(1), 11-23. doi: http://dx.doi.org/10.7203/REK.1.1.7947

Cortina, Adela (1985). Razón comunicativa y responsabilidad solidaria. Salamanca: Sigueme.

Cortina, Adela (1986). Ética mínima. Madrid: Tecnos.

Cortina, Adela (1997). Ciudadanos del mundo. Hacia una teoría de la ciudadanía. Madrid: Alianza Editorial.

Cortina, Adela (2007). Ética de la razón cordial. Educar en la ciudadanía. Oviedo: Ediciones Nobel.

Cortina, Adela (2009). Las fronteras de la persona: el valor de los animales, la dignidad de los humanos. Madrid: Taurus.

Cortina, Adela (2010). Justicia Cordial. Madrid: Trotta.

Cortina, Adela y Conill, Jesús (1989). Estudio preliminar. En Kant (1797). La Metafísica de las Costumbres. Madrid: Tecnos.

Cortina, Adela, Conill, Jesús y García-Marzá, Domingo (eds.) (2008). Public Reason and Applied Ethics: The Ways of Practical Reason in a Pluralist Society. Hampshire: Ashgate Publishing.

Council for International Organizations of Medical Sciences in collaboration with the World Health Organization (2016). International Ethical Guidelines for Health-related Research Involving Humans. Biomedical Research. Recuperado de: http://www.sciencedirect.com/science/article/B6V C6 $45 \mathrm{~F}_{5} \mathrm{Xo2}$ 9C/2/e44bc37a6e392634b1cf 436105978 fo1

Cragoe, Nicholas (2019). Oversight: Community vulnerabilities in the blind spot of research ethics. Research Ethics, 15(2), 1-15. doi: http://dx.doi.org/10. 1177/1747016117739936

Darwall, Stephen (1977). Two Kinds of Respect. Ethics, 88(1), 36-49.

Dávila, Johnny (2011). Algunas consideraciones sobre el sentimiento de respeto a la ley moral. Discusiones Filosóficas, 12(18), 145-154. 
Dingwall, Robert, Iphofen, Ron, Lewis, Janet, Oates, John y Emmerich, Nathan (2017). Towards Common Principles for Social Science Research Ethics: A Discussion Document for the Academy of Social Sciences. Finding Common Ground: Consensus in Research Ethics Across the Social Sciences (Advances in Research Ethics and Integrity, vol. 1), 111-123. doi: http://dx.doi.org/10.1108/s2398-601820170000001010

Dittmer, Cordula y Lorenz, Daniel (2018). Forschen im Kontext von Vulnerabilität und extremem Leid - Ethische Fragen der sozialwissenschaftlichen Katastrophenforschung 1. Katastrophenforschung: Eine szenische Darstellung, 19(3).

Erken, Arthur (2020). Against my will. Defying the practices that harm women and girls and undermine equality. State of World Population 2020. New York: UNFPA.

European Commission (2018). Ethics in Social Science and Humanities. Recuperado de: https://ec.europa.eu/info/sites/default/files/6._h2ozo_ethics-socscience-humanities_en.pdf

Ezekiel, J. Emanuel y Weijer, Charles (2005). Protecting Communities in Research: From a New Principle to Rational Protections. Belmont Revisited: Ethical Principles for Research with Human Subjects.

Giorgini, Giovanni e Irrer, Elena (2017). Roots fo respect. A Historic-Philosophical Itinerary. Berlin: De Gruyter.

Glasius, Marlies, Lange, Meta De, Bartman, Joss, Dalmasso, Emmanuela Lv, Aofei, Sordi, Adele Del, Michaelsen, Marcus y Ruijgrok, Kris (2018). Research, ethics and Risk in the authoritarian field. Cham: Palgrave Macmillan.

Göbel, Marie (2017). Respect as the Foundation of Human Rights: To What Extent Can This View Be Attributed to Kant? En Giorgini, Giovanni e Irrera, Elena (ed.). Roots of Respect (149-170). Berlin: De Gruyter.

Habermas, Jürgen (1968). Erkenntnis Und Interesse. Felix Meiner Verlag.

Habermas, Jürgen (1983). Moralbewusstsein und Kommunikatives Handeln. Frankfurt am Main: Shurkamp.

Kant, Immanuel (1785). Grundlegung zur Metaphysik der Sitten. Frankfurt am Main: Shurkamp.

Kant, Immanuel (1797). La metafísica de las costumbres. Madrid: Tecnos.

Kipnis, Kenneth (2001). Vulnerability in Research Subjects: A Bioethical Taxonomy. En Ethical and Policy Issues in Research Involving Human Participants, vol. 1, (576-580). Maryland. doi: http://dx.doi.org/10.1159/oooo55866 Korsgaard, Christine (2009). Self-Constitution. Agency, Identity, and Integrity. Oxford: Oxford University Press. 
Lozano, J. Félix y Escrich, Teresa (2017). Cultural Diversity in Business: A Critical Reflection on the Ideology of Tolerance. Journal of Business Ethics, 142(4), 679-696. doi: http://dx.doi.org/10.1007/s10551-016-3113-y.

Marcuse, Herbert (1965). Repressive Toleranz. Kritik Der Reinen Toleranz, 91128.

Milgram, Stanley (1974). Obedience to authority: An experimental view. New York: Harper and Row.

Morris, Nina (2015). Providing ethical guidance for collaborative research in developing countries. Research Ethics. doi: http://dx.doi.org/10.1177/1747016115 586759

Nussbaum, Martha (2000). Women and Human Development. Chicago: Cambridge University Press. doi: http://dx.doi.org/10.1017/cbo9780511841286

Schadow, Steffi (2015). Achtung für das Gesetz. Moral und Motivation bei Kant. Kant Studien, 106(1), 160-164. doi: http://dx.doi.org/10.1515/kant-20150017

Schroeder, Doris (2008). Dignity: Two riddles and four concepts. Cambridge Quarterly of Healthcare Ethics, $17(2), 230-238$. doi: http://dx.doi.org/10.1017/S 0963180108080262

Schroeder, Doris y Bani-Sadr, Abol-hassan (2017). Dignity in the 21st Century Middle East and West SpringerBriefs in Philosophy. Recuperado de: https://link springer com.ezproxy.puc.cl/content/pdf/10.1007\%2F978-3319-58020-3.pdf

Schroeder, Doris, Chatfield, Kate, Muthuswamy, Vasanta y Kumar, Nandini (2020). Ethics Dumping - How not to do research in resource-poor settings. Academics Stand Against Poverty, 1(1), 32-54.

Schroeder, Doris, Chatfield, Kate, Singh, Michelle, Chennells, Roger y Herissone-Kelly, Peter (2019). Equitable Research Partnerships. A Global Code of Conduct to Counter Ethics Dumping. Cham: Springer. Recuperado de: https://link.springer.com/book/10.1007\%2F978-3-030-15745-6

Schroeder, Doris, Cook, Julie, Hirsch, François y Fenet, Solveig (2018). Ethics Dumping Case Studies from North-South Research. Cham: Springer.

Schroeder, Doris y Gefenas, Eugenijus (2009). Vulnerability: Too vague and too broad? Cambridge Quarterly of Healthcare Ethics, 18(2), 113-121. doi: http://dx.doi.org/10.1017/Sog63180109090203

Sen, Amartya (1999). Desarrollo y Libertad. Barcelona: Planeta.

Sen, Amartya (2006). Identity and Violence. New York: W. W. Norton and Company.

Sensen, Oliver (2011). Kant on human dignity. Göttingen: Walter de Gruyter. 
Spitz, Vivien (2005). Doctors from Hell. Boulder: Sentien Publications.

Torralba, José Maria (2020). El sentimiento de respeto y la estructura del vínculo moral. Revista de Estudios Kantianos, 5(1), 174-191. doi: http://dx.doi.org/10.7203/REK.1.1.7947

Zimbardo, Philip (2007). The Lucifer Effect: Understanding how good people turn evil. New York: Random House. 